\title{
$\beta$-hairpin Crowding Agents Affect Alpha Helix Stability in Crowded Environments
}

\author{
Bryanne Macdonald, Shannon McCarley ${ }^{\dagger}$, Sundus Noeen ${ }^{\dagger}$, and Alan van Giessen* \\ Department of Chemistry \\ Mount Holyoke College \\ South Hadley, MA 01075 \\ $\dagger$ These authors contributed equally.
}


Figure S1. The average distance between the terminal $\alpha$-carbons (end-to-end distance) as a function of temperature for the seven different crowder systems. The simulation box has side lengths of $65 \AA$ and the longest-range interaction in the protein model is $10 \AA$. Colors are the same as in Figure 2.

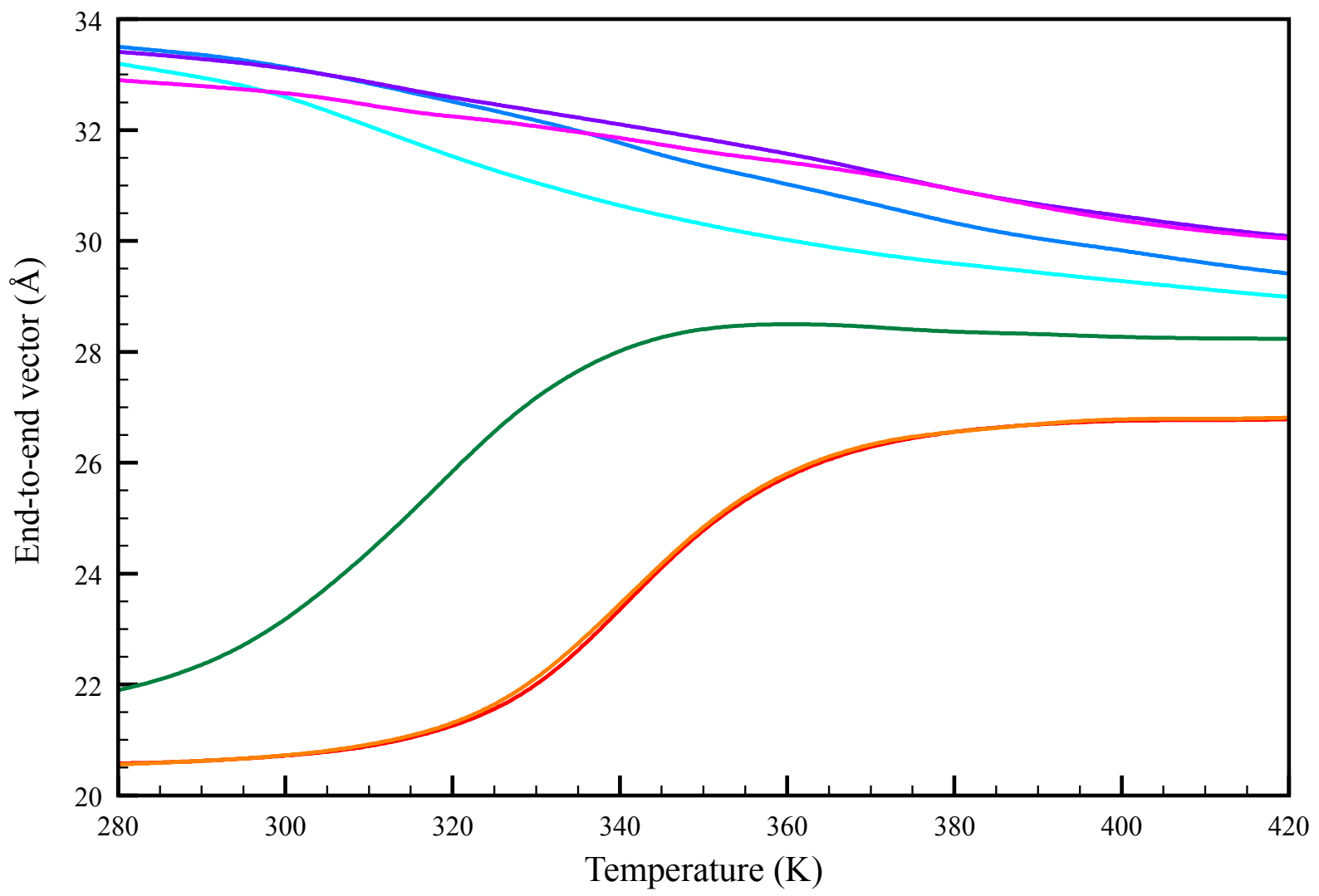


Figure S2. The hydrogen bond energy for the (AAQAA) $)_{3}$ test protein (TP) in the presence of serine crowding agents (top) and histidine crowding agents (bottom). Note that the crowding agent hydrogen bond energy for serine crowders is essentially constant throughout the entire temperature range, indicating that intermolecular hydrogen bonding plays little role in the destabilization of the test protein. The hydrogen bond energies for the test protein and the crowding agents are equal for the histidine crowders, indicating that nearly all the hydrogen bonds are between the test protein and a crowding agents and there are very few test protein-test protein hydrogen bonds.

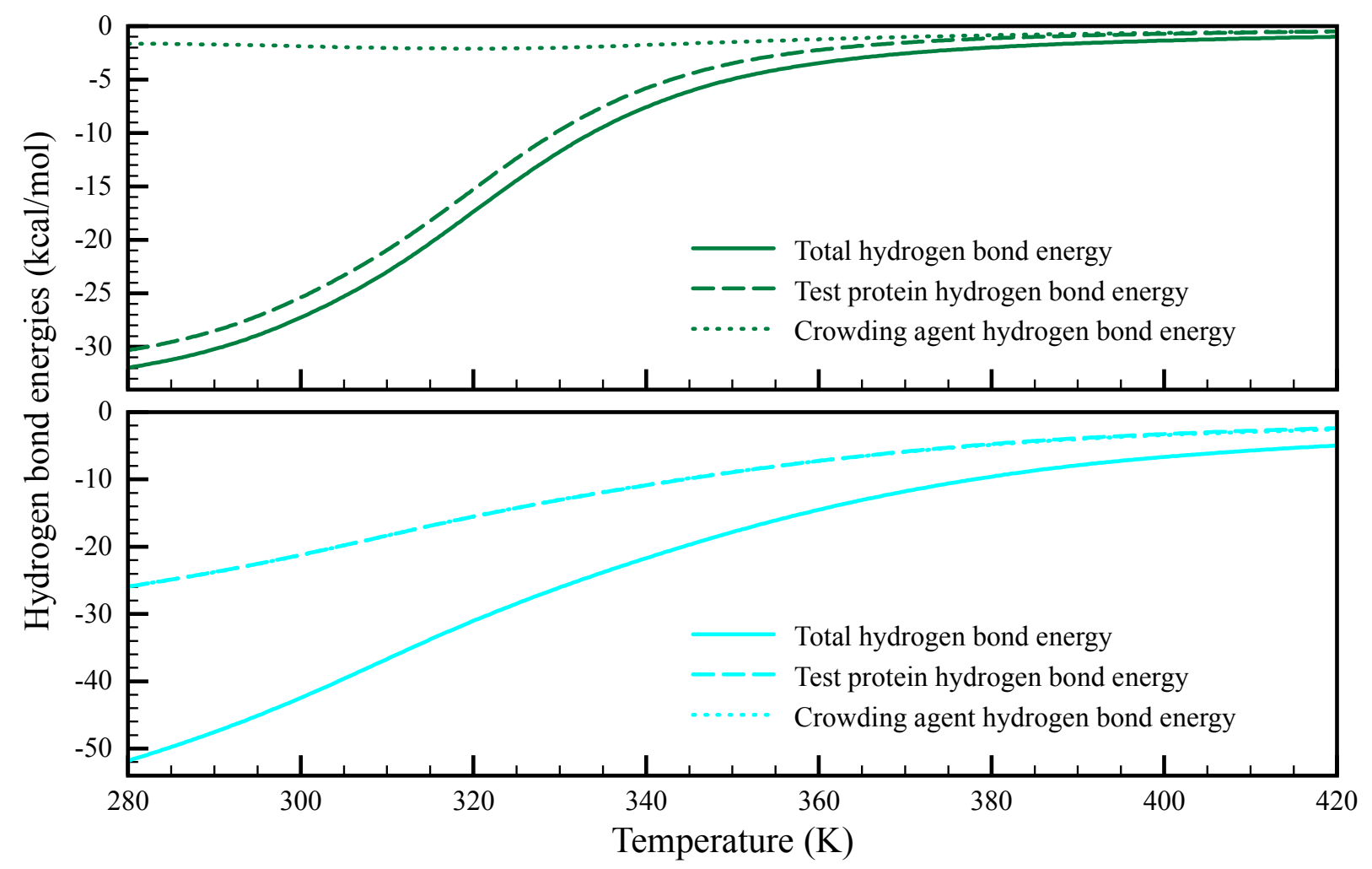


Figure S3. The free energy along the minimum path $\xi$ from the native state to the extended state across each free energy surface relative to the global minimum. $\xi=0$ in the lower left-hand corner of the surface and $\xi=1$ in the upper right-hand corner. The colors are the same as in Figure 2.

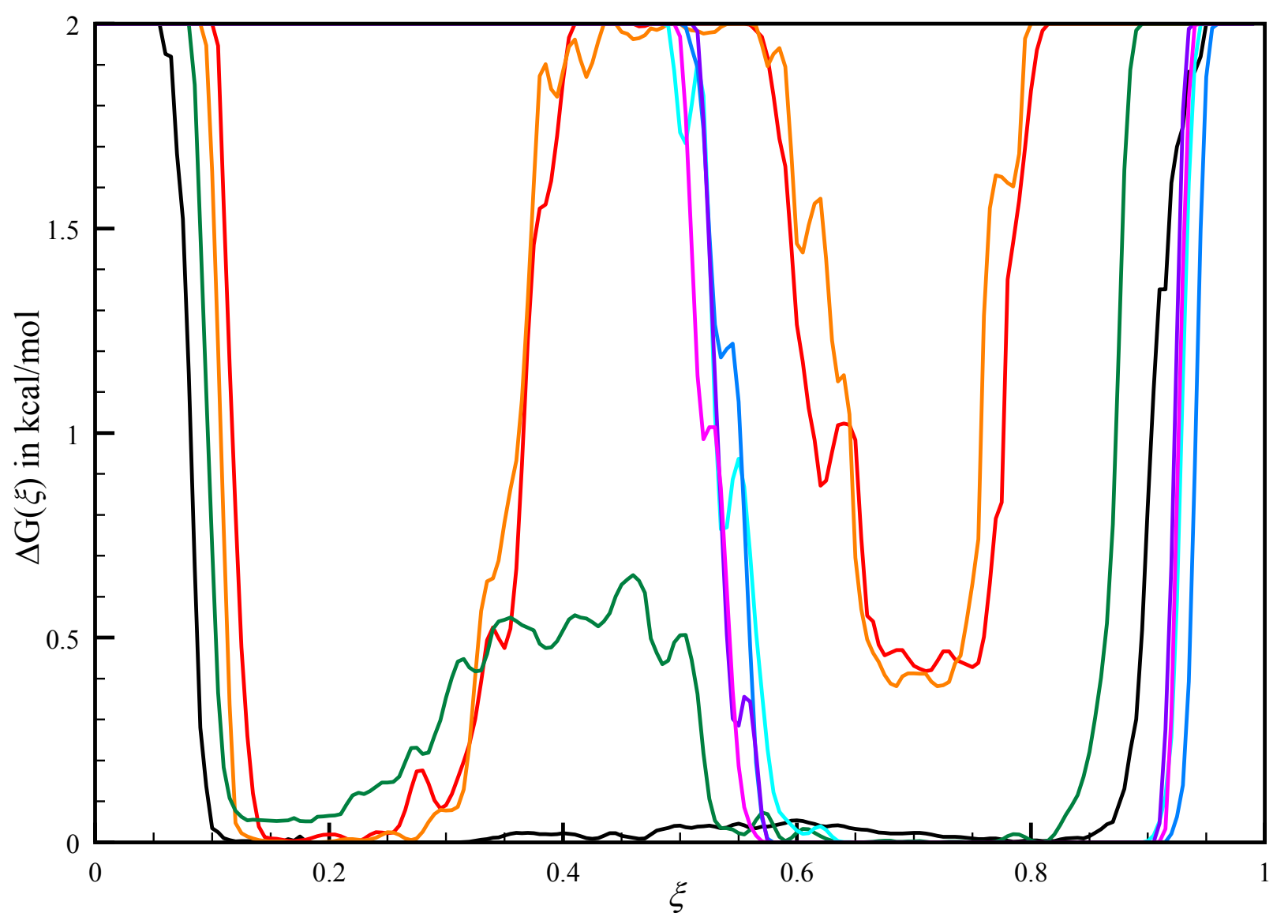


Figure S4. Representative configurations of the test protein and leucine crowding agents at $T=$ $322.4 \mathrm{~K}$. The test protein is shown in red. In the top row, the test protein has one $\beta$-segment while in the bottom row it has two. Configurations were made using VMD (Humphrey, W.; Dalke, A.; Schulten, K. VMD - Visual Molecular Dynamics. J. Molec. Graphics. 1996, 4, 3338).
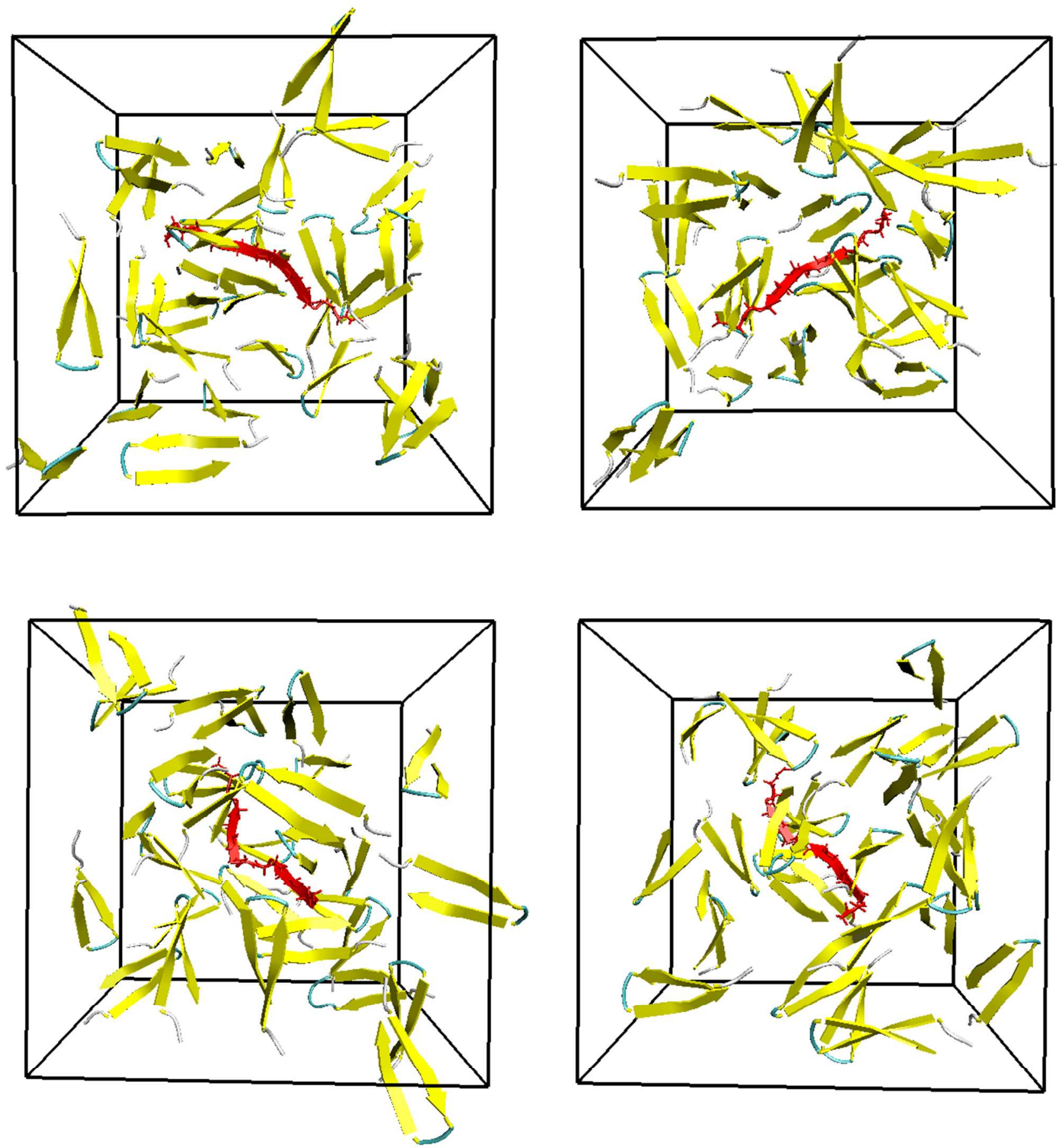
Figure S5. Potential energy surface of the monomer in dilute solution in $\mathrm{kcal} / \mathrm{mol}$ as a function of the radius of gyration and the parameter $Q_{\psi}$. The native state is in the lower left (dark blue). The left-handed $\alpha$-helix is indicated by the arrow.

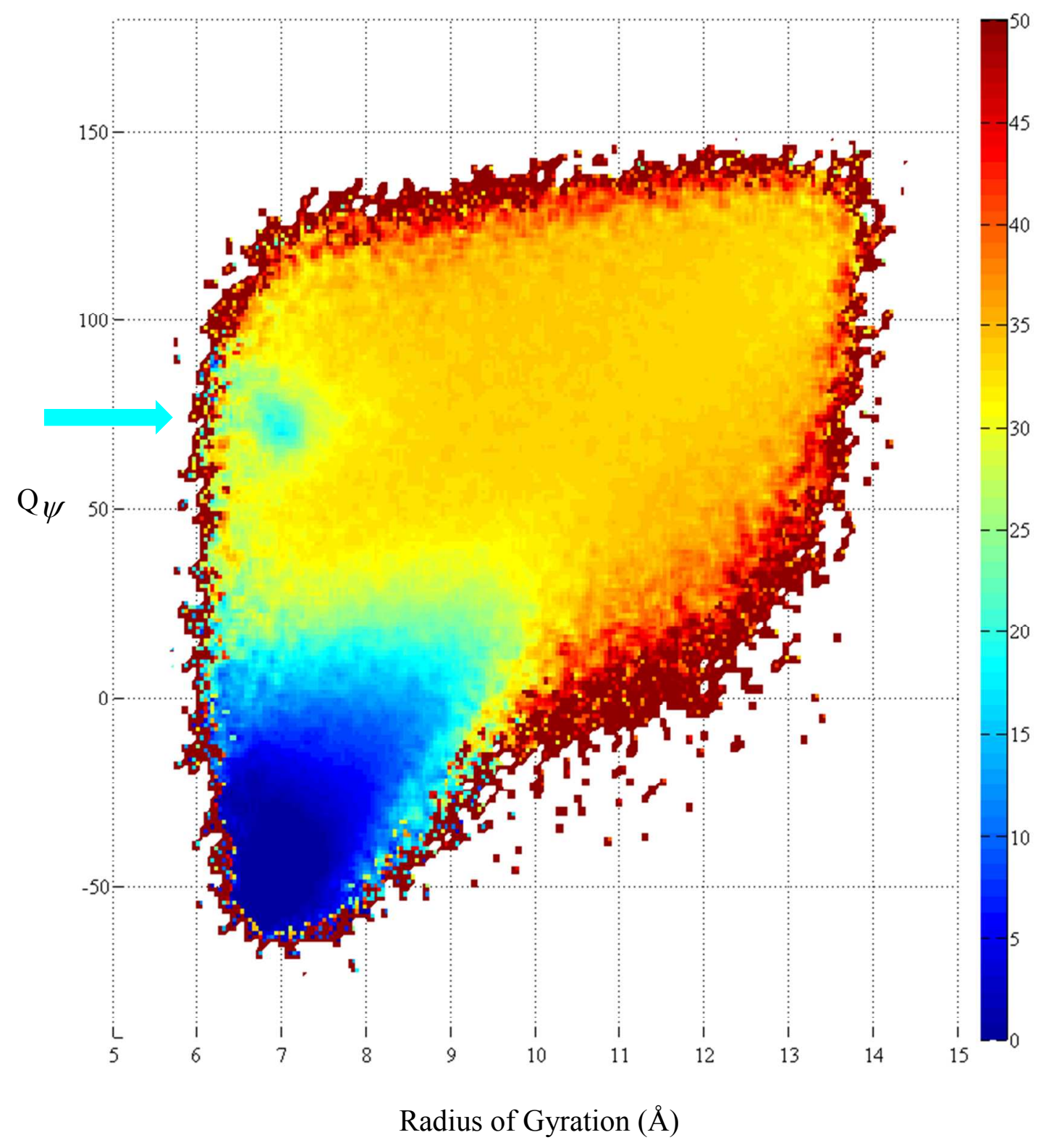

